\title{
OSTEOSARCOMA LUMBOSACRO: PRESENTACION DE UN CASO. APORTE DE LA MEDICINA NUCLEAR Y OTRAS TECNICAS DE IMAGEN
}

Drs. Carolina Aqueveque $A^{(1)}$, Teresa Massardo $V^{(1)}$, Marcela Muñoz M, Luis Velozo $P^{(2)}$.

1. Hospital Clínico de la Universidad de Chile.

2. Hospital Roberto del Río, Santiago. Chile.

\begin{abstract}
A 12 year-old-girl, with 11 months of natural evolution of a conventional osteosarcoma Stage IV in pelvis, with bilateral sacral root involvement, clinically manifested with paraparesia, neurogenic bladder and transverse myelitis. The images (pelvic and thoracic CT, pelvic MRI and bone scintigraphy), allowed correct staging, leading to choose the most adequate therapy. We discuss the diagnostic imaging with emphasis in bone scans. Currently, the patient is under palliative chemotherapy.
\end{abstract}

Key words: Bone scintigraphy, Fluor ${ }^{18_{-}}$ desoxyglucose, Malignant bone tumor, Osteosarcoma, PET.

Resumen: Se presenta el caso de una escolar, de sexo femenino, de 12 años de edad, con un osteosarcoma convencional de pelvis estadio IV, que evoluciona en forma natural por 11 meses, con compromiso bilateral de las raíces sacras y que se manifiesta clínicamente como paraparesia, vejiga neurogénica y mielitis transversa. El estudio de imágenes tanto anatómico como funcional (TC de tórax y pelvis, RM de pelvis y cintigrama óseo), permitió su correcta estadificación y selección de quimioterapia paliativa. Se analiza el caso desde el punto de vista de las imágenes destacando el aporte de la medicina nuclear.

Palabras claves: Cintigrama óseo, Fluor ${ }^{18}$ deoxiglucosa, Osteosarcoma, PET, Tumores óseos malignos.

Aqueveque C. y cols. Osteosarcoma lumbosacro: Presentación de un caso. Aporte de la medicina nuclear y otras técnicas de imagen. Rev Chil Radiol 2003; 9:206-210. Correspondencia: Dra. Carolina Aqueveque A.

Sección Medicina Nuclear. Departamento de Medicina, Hospital Clínico Universidad de Chile

Santos Dumont 999, 1er piso, sector E.

Fonos: 777 0569, 687 8184. FAX: 7777618

caqueveque@machi.med.uchile.cl
Introducción

Los estudios de imágenes en pacientes con patología tumoral ósea, se pueden dividir en anatómicos o estructurales y funcionales 0 metabólicos. Entre los primeros están la Radiología simple (Rx simple), la Tomografía Computada (TC) y la Resonancia Magnética (RM). En el segundo grupo están el Cintigrama óseo (CO) planar o SPECT y la Tomografía por Emisión de Positrones (PET).

Ante la sospecha de una lesión ósea, la Rx simple es la aproximación inicial. El compromiso óseo solo será evidente si el hueso afectado ha sufrido una pérdida mineral del 30 a $50 \%{ }^{(1)}$.

Tanto la TC como la RM son técnicas indispensables para obtener con precisión la ubicación, tamaño y forma de un tumor, además de evaluar su relación con los tejidos adyacentes ${ }^{(2)}$. Estas técnicas anatómicas son complementarias con el CO, para la evaluación de la invasión tumoral local y a distancia en el esqueleto y para planificar el procedimiento quirúrgico. Este último tiene una alta sensibilidad para detectar aumentos focales o difusos de la actividad osteoblástica, independiente de la causa que la origina. La infección, inflamación o fracturas, y también la reacción a la infiltración ósea por células tumorales provenientes de neoplasias benignas o malignas, primarias o metastásicas dan focos de captación aumentada ${ }^{(1)}$. Las ventajas del CO, son su bajo costo y baja dosis de radiación, considerando que se trata de un estudio del cuerpo entero. Como desventaja se reconoce su baja especificidad y la sobreestimación del tamaño tumoral, en aquellas lesiones con intensa captación ${ }^{(2)}$. Su principal indicación en oncología es para definir la extensión local y a distancia de los tumores óseos, ello es importante ya que la detección de metástasis osteoblásticas, cambia el estadiaje, determinando el pronóstico y orientando al clínico en la elección del tratamiento en diversas neoplasias (mama, próstata y pulmón, entre otras) $)^{(1,3,4)}$. 
La introducción del PET nos permitió disponer de una técnica para evaluar el metabolismo glucídico en tumores de alto grado o de alto recambio celular. Usa como trazador la molécula de deoxiglucosa marcada con Flúor $^{18}$ (FDG), la que queda atrapada en el espacio intracelular; el $\mathrm{F}^{18}$ tiene una vida media de 2 horas y la FDG presenta eliminación vía renal.

En osteosarcoma, las imágenes del CO no tienen valor pronóstico, ni pueden evaluar con exactitud la respuesta a tratamiento, ya sea quirúrgico, con quimioterapia (QT) o radioterapia (RT). Los estudios metabólicos por emisión de positrones, tendrían valor como predictor de sobrevida al momento del diagnóstico, según la intensidad de captación del FDG ${ }^{(6)}$ y sería un método eficaz para clasificar a los pacientes sometidos a QT prequirúrgica en respondedores y no respondedores ${ }^{(7)}$.

Entre las neoplasias malignas pediátricas, los tumores óseos primarios, para el año 2000, ocupaban el séptimo lugar en orden de frecuencia en EEUU. EI más común es el osteosarcoma, seguido por el sarcoma de Ewing y en menor frecuencia el condrosarcoma ${ }^{(8)}$. El osteosarcoma afecta principalmente a adolescentes y adultos jóvenes menores de 30 años; se ubica preferentemente en las metáfisis de los huesos largos especialmente fémur, tibia y húmero. El sarcoma de Ewing compromete principalmente pelvis y la región metáfisis/diáfisis de huesos largos tales como fémur, costillas y tibia ${ }^{(2)}$.

La incidencia de tumores óseos malignos en EEUU entre 1975 y 2000 fue de 8.5 por 1.000 .000 en niños de 1 a 19 años, con una sobrevida promedio a 5 años de $65.3 \%$ en ambos sexos ${ }^{(8)}$. No contamos con estos valores específicos en el ámbito nacional.

\section{Caso clínico}

Escolar de 12 años 10 meses, de sexo femenino, sin antecedentes mórbidos de importancia. Presenta

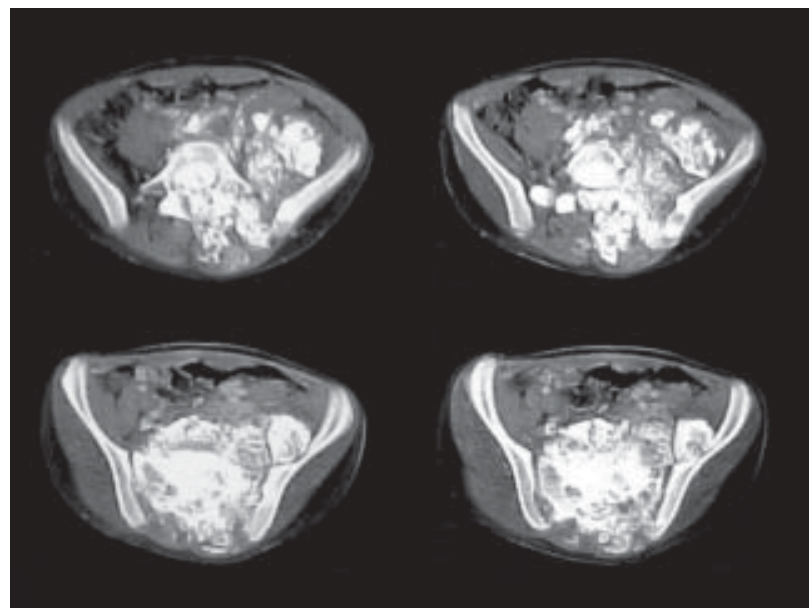

Figura 1. TC de pelvis con ventana ósea, cortes axiales desde L5 a distal. un cuadro de 9 meses de evolución, caracterizado por dolor y parestesias progresivas en extremidad inferior izquierda, palpitaciones, ahogo, labilidad emocional e inapetencia. Evoluciona con baja de peso, claudicación de la marcha por dolor, disminución de la fuerza e incontinencia urinaria.

Clínicamente se indicó rehabilitación motora, durante la cual se constata por electromiografía la presencia de polineuropatía crónica, vejiga neurogénica y mielitis transversa. Evoluciona con paraplejia de extremidades inferiores, momento en el cual es sometida a estudio de imágenes, tanto anatómico como funcional: TC y RM de pelvis más CO.

Los análisis de laboratorio mostraron anemia leve normocítica normocrómica, fosfatasas alcalinas aumentadas al triple de su valor normal, con función hepática y renal normales.

La TC lumbosacra demostró un extenso proceso expansivo tumoral de aproximadamente $15 \mathrm{~cm}$ de eje mayor en región lumbosacra, lateralizado a izquierda, con neoformación ósea de contornos irregulares que infiltra el psoas y musculatura de la pelvis. La extensión hacia la región prevertebral en ambos lados, produce rechazo del recto hacia anterior. Hay además compromiso del plexo sacro y del canal dural lumbar bajo (Figura 1). Las reconstrucciones 3D demuestran las extensas zonas de neoformación ósea, destrucción del esqueleto pelviano (Figura 2) y la relación con los vasos femorales que atraviesan la lesión (Figura 3).

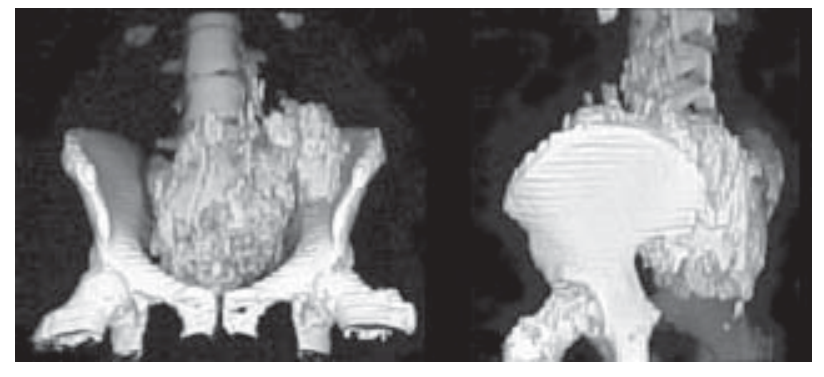

Figura 2. TC de pelvis, reconstrucción en $3 D$ AP y OPI, con ventana ósea.

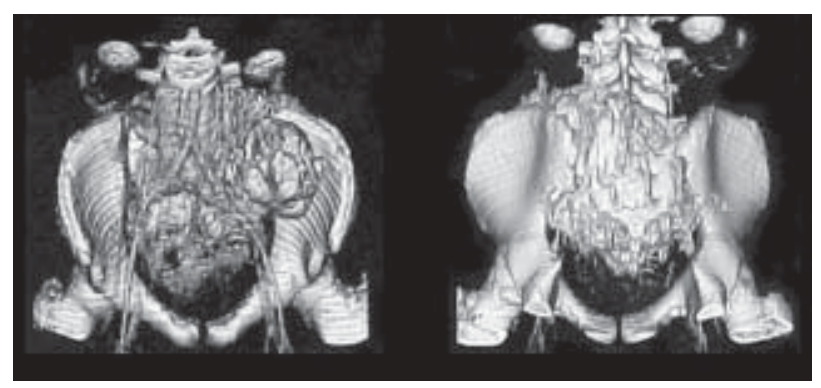

Figura 3. TC de pelvis, reconstrucción en $3 D A P$ y $P A$, con ventana para partes blandas. 
La RM de región lumbosacra y pelvis mostró que la lesión compromete toda la región del sacro, con ocupación completa del canal dural desde L4 hacia caudal, destrucción de los arcos posteriores de todos los segmentos sacros más los dos últimos segmentos lumbares y extensión hacia la región prevertebral, lateralizado a izquierda. Ocupación pelviana, infiltración del psoas, rechazo de recto y de la pared posterior de la vejiga y probable englobamiento de la aorta y vasos femorales (Figura 4).

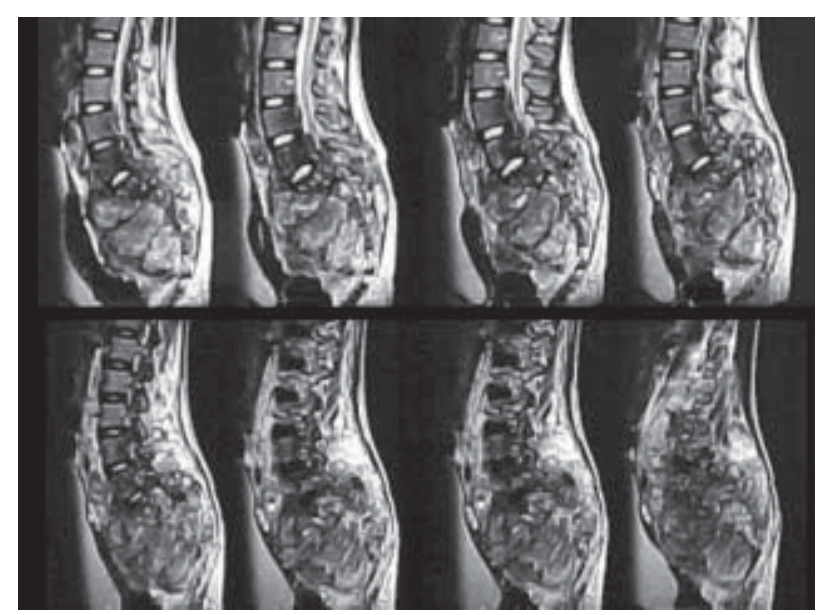

Figura 4. RM secuencia T2 de columna vertebral dorsolumbar; cortes sagitales de derecha a izquierda.

En el cintigrama óseo $T c^{99 m}-M D P$ en imágenes AP y PA segmentarias de cuerpo entero se visualizó un intenso aumento de la actividad osteoblástica en sacro, con alteración de su morfología por protrusión hacia posterior, anterior y superior izquierdo. La captación del radiofárrmaco es muy irregular, compromete cóccix, articulación sacroilíaca izquierda y región superior de ala ilíaca izquierda, con mayor intensidad a ese nivel (Figura 5). En columna lumbar se evidencia intensa captación en cuerpo de L5, la que se extiende hacia lateral izquierdo, y hemicuerpo izquierdo de L4 (Figura 6). No hay evidencia de focos metastásicos a distancia (Figuras 5 y 7).

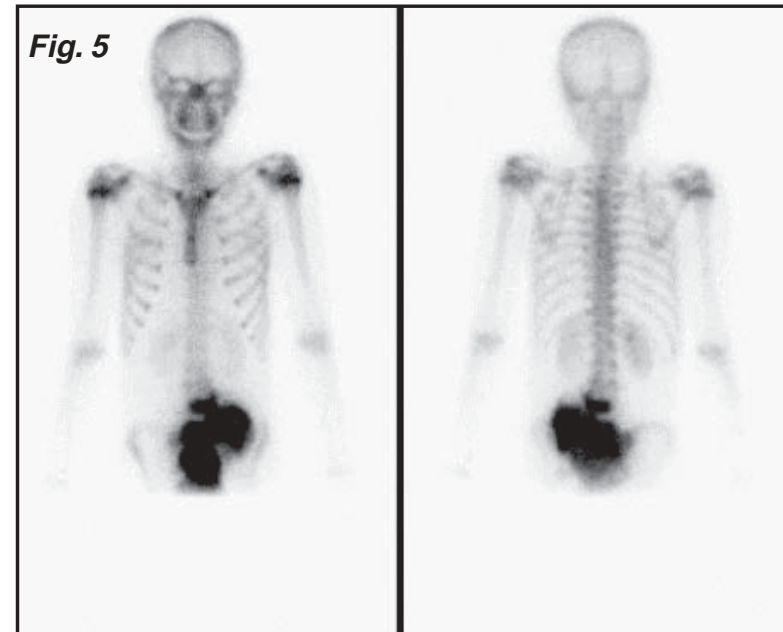

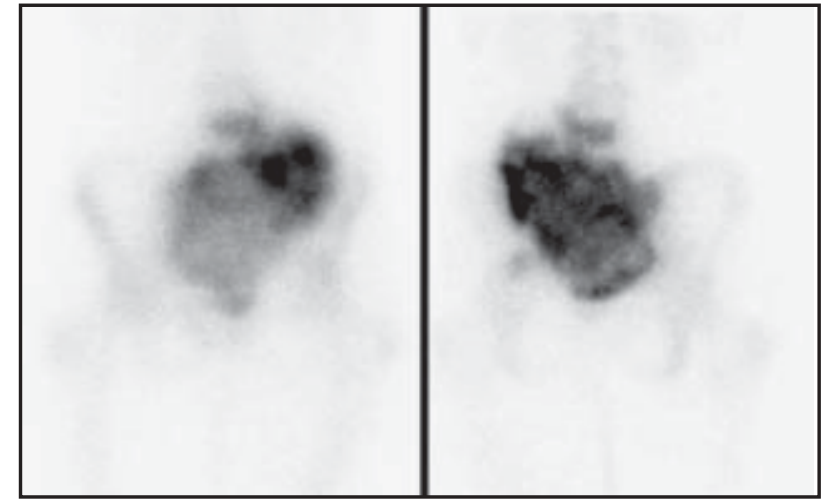

Figura 6. CO, imágenes segmentarias de pelvis en proyección AP y $P A$.

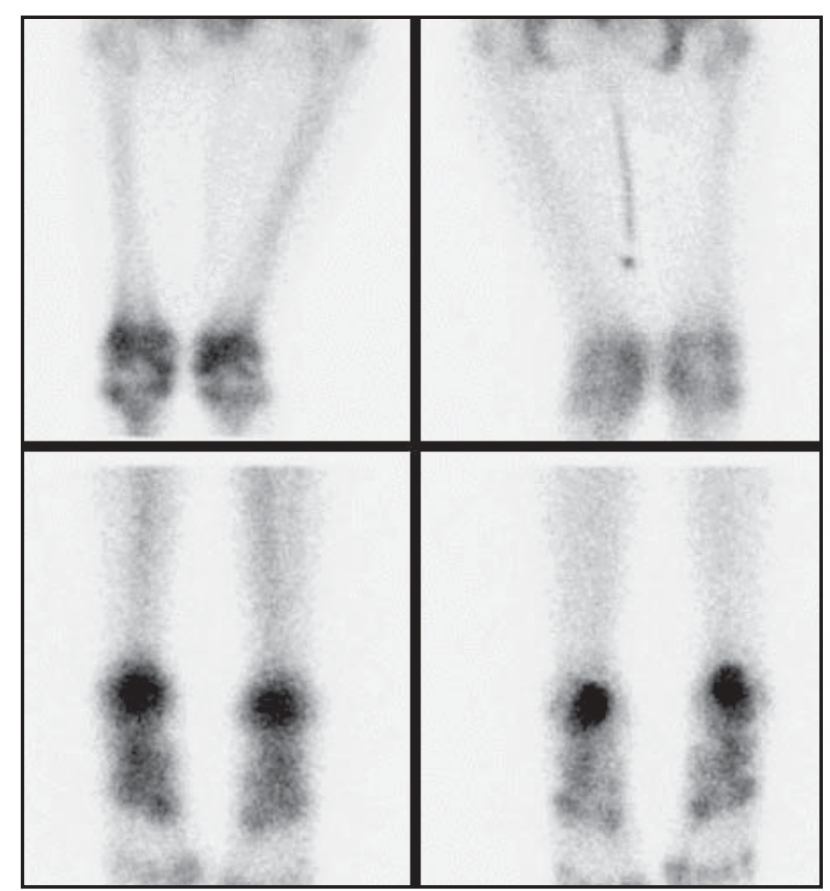

Figura 7. CO imagen segmentaria de muslos, piernas y pies en proyección AP y PA. Se observa intensa captación en cartílagos de crecimiento, lo que es normal para la edad de la paciente.

Figura 5. CO en proyección AP y PA de esqueleto axial. Las imágenes de cuerpo entero no fueron completadas, por intenso dolor sacro de la paciente, que le impiden mantener la posición decúbito.

Se efectuó biopsia incisional, lográndose la exéresis de aproximadamente $30 \%$ de la masa tumoral sacra, con descompresión del canal raquídeo. El informe anátomo-patológico concluyó: Osteosarcoma convencional, con extensa diferenciación condroblástica y con bordes positivos en todas las muestras.

La TC de tórax efectuada posterior a la cirugía demostró 4 pequeñas imágenes nodulares densas 
sugerentes de localización secundaria pulmonar bilateral. Actualmente, la paciente está en quimioterapia paliativa.

\section{Discusión}

Es bien conocido que a pesar de la alta sensibilidad del $\mathrm{CO}$, es un examen relativamente inespecífico. Los hallazgos de la RM y TC, son muy importantes para caracterizar la lesión, sin embargo, el diagnóstico definitivo es histológico. En el presente caso, la TC y RM fueron sugerentes de tumor maligno. Por tratarse de una lesión única, de gran extensión, en pelvis y de comportamiento infiltrante de tejidos blandos (2), las características cintigráficas en cuanto a intensidad y tamaño fueron compatibles con tumor óseo primario, probablemente maligno. Lo más orientador es la tendencia a infiltrar el hueso afectado en forma difusa y su alta actividad osteoblástica, reflejada en la gran captación de $\mathrm{Tc}^{99 \mathrm{~m}}$-MDP, con ruptura de la cortical e invasión a tejidos blandos. No se evidenciaron lesiones a distancia.

El osteosarcoma se caracteriza histológicamente por formación de tejido osteoide, infiltrante, donde la lesión primaria y sus metástasis pueden potencialmente captar el radiofármaco y así aparecer focos hipercaptantes en tejidos blandos adyacentes al foco primario y/o órganos a distancia, principalmente pulmón. El sarcoma de Ewing se presenta cintigráficamente como un aumento de la captación focal, intensa y homogénea, con límites poco definidos, moderada distorsión ósea, sin destrucción de la cortical, sin evidencias cintigráficas de invasión a partes blandas. El condrosarcoma, más propio de adultos entre 30 y 60 años, en su variedad exofítica habría mostrado áreas moteadas de actividad osteoblástica moderadamente aumentada, sobre una actividad de fondo fría o hipercaptación focal intensa, pudiendo ser infiltrante ${ }^{(4)}$.

La ubicación en pelvis del tumor presentado, plantea algunos diagnósticos diferenciales, como primera etiología el sarcoma de Ewing. Un osteosarcoma puede afectar la pelvis, pero con menor frecuencia que a los huesos largos. Menos probable, es que se trate de un tumor de células gigantes, que es más frecuente entre los 20 y 40 años, que generalmente afecta los huesos largos de extremidades inferiores y prácticamente puede ser descartado si no compromete el extremo articular del hueso(2). Los hallazgos de imágenes y la edad de la paciente sugieren osteosarcoma, sin poder descartar condrosarcoma, aunque con baja probabilidad.

Las metástasis pulmonares en esta paciente no fueron evidentes al CO. En la literatura, se ha analizado el beneficio de complementar el $\mathrm{CO}$ con SPECT de tórax, lo que se comparó con TC tórax y se concluyó que un SPECT negativo no descarta metástasis ${ }^{(9)}$. La TC es la primera elección para diagnóstico de compromiso pulmonar secundario.

En el seguimiento de metástasis pulmonares, el aporte del CO es menor ${ }^{(10,11)}$. El PET-FDG tiene utilidad limitada para pesquisarlas ${ }^{(12)}$. Algunos autores emplean el estudio de cuerpo entero con Talio 201 o Sestamibi-Tc99m, que también son marcadores tumorales, con buen rendimiento para detección de metástasis. Estos marcadores están, en evaluación para predicción de respuesta a terapia ${ }^{(13-16)}$.

Franzius et al. ${ }^{(6)}$ plantean que el PET-FDG- ${ }^{18}$ realizado al momento del diagnóstico de osteosarcoma, aporta valor pronóstico dependiendo del grado de aumento del metabolismo medida como captación máxima de FDG- ${ }^{18} \mathrm{~F}$ del tumor con relación a la misma zona contralateral sana. Otros trabajos han confirmado que en sarcomas óseos pediátricos, el PET-FDG- $\mathrm{F}^{18}$ también es útil para discriminar entre respondedores y no respondedores a quimioterapia prequirúrgica ${ }^{(7)}$. Según una revisión reciente del tema, habría situaciones clínicas en las cuales hacer un PETFGD-F ${ }^{18}$ es de utilidad para el manejo del paciente con osteosarcoma ${ }^{(12)}$ para: a) Predecir la evolución según el grado de respuesta a la quimioterapia. b) Diferenciar cambios postquirúrgicos de tejido tumoral o recaída local. c) Detectar diseminación hematógena durante el seguimiento en imágenes de cuerpo entero. En el caso de masas pulmonares, el PET sería útil para diferenciar entre lesiones benignas de metastásicas.

Concluyendo, en el estudio previo al tratamiento de un osteosarcoma, el predictor de sobrevida más fuerte es la presencia o ausencia de metástasis; el $\mathrm{CO}$ es el mejor método para su detección a nivel esquelético, por su capacidad de realizar análisis de la actividad osteoblástica normal en cuerpo entero. Las imágenes estructurales son básicas para caracterizar el tumor primario y su extensión local siendo complementarias con el anterior. Idealmente, la respuesta a la quimioterapia podría ser evaluada con PET-FDG.

\section{Bibliografía}

1. Lovera C, Guzman G, Batista JF, Solano ME. Cap 6.2: Gammagrafía ósea en el estudio de la patología ósea tumoral. pp 341-347. Roca I, Avila E, Coll C, González P. Cap 6.7: Sistema musculoesquelético en pediatría. pp 391-408. En "Medicina Nuclear Aplicaciones Clínicas". Carrió y González. Eds. Masson 2003.

2. Greinspan. Remagen "Tumores óseos y articulaciones". Ed. Marban 2002.Cap 1: Radiologic and Pathologic Approach to Bone Tumors, pp 1-21.

3. Pauwels EK, Schutze HE, Arndt JW. Scintigraphic detection of bone metastasis. In: Winkler CG, ed. Nuclear Medicine in Clinical Oncology. Bonn; Society of Nuclear Medicine Europe 1985; 42-43. 
4. Fred A. Metter Jr. "Radionuclide Bone Imaging And Densitometry". Churchill Livingstone.1988. Cap 4: The radionuclide bone scan in malignant disease. p 63-86. Cap 8: Pediatric applications of radionuclide bone imaging. pp 161-195.

5. Subramanian G, McAfee J, Blair R. et al. Technetium 99m Methylene Diphosphonate a superior agent for skeletal imaging: comparison with other technetium complexes. J Nucl Med 1975; 8: 744-755.

6. Franzius $\mathrm{C}$, Bielack S, Flege $\mathrm{S}$, et al. Prognostic signifcance of 18F-FDG and 99mTc-Methylene Diphosphonate uptake in primary osteosarcoma. J Nucl Med 2002; 43: 1012-1017.

7. Hawkins DS, Rajendran JG, Conrad EU. $3^{\text {rd }}$ et al. Evaluation of chemotherapy response in pediatric bone sarcomas by [F-18]-fluorodeoxy-D-glucose positron emission tomography. Cancer 2002; 94: 3277-3284. Erratum in Cancer 2003; 195; 97: 3130.

8. Ries LAG, Eisner MP, Kosary CL, et al. SEER Cancer Statistics Review, 1975-2000, National Cancer Institute. Bethesda, MD, http://seer.cancer.gov/csr/1975-2000, 2003.

9. Pevarski DJ, Drane WE, Scarborough MT. The usefulness of bone scintigraphy with SPECT images for detection of pulmonary metastases from osteosarcoma. Am J Roentgenol 1998;170:319-322.

10. Rees CR, Siddiqui $A R$, duCret $R$. The role of bone scintigraphy in osteogenic sarcoma. Skeletal Radiol 1986;15:365-367.

11. Korholz D, Wirtz I, Vosberg $\mathrm{H}$ et al. The role of bone scintigraphy in the follow-up of osteogenic sarcoma. Eur J Cancer 1996; 32A:461-464.

12. Brenner W, Bohuslavizki KH, Eary JF. PET Imaging of Osteosarcoma. J Nucl Med 2003; 44:930-942.

13. Kaste SC, Billips $C$, Tan M, et al. Thallium bone imaging as an indicator of response and outcome in nonmetastatic primary extremity osteosarcoma. Pediatr Radiol 2001; 31:251-6.

14. Goto Y, Ihara K, Kawauchi S, et al. Clinical significance of Thallium-201 scintigraphy in bone and soft tissue tumors. J Orthop Sci 2002; 7:304-312.

15. Burak Z, Moretti JL, Ersoy O, et al. 99mTc-MIBI imaging as a predictor of therapy response in osteosarcoma compared with multidrug resistance-associated protein and P-Glycoprotein expression. J Nucl Med 2003; 44:1394-1401.

16. Imbriaco $M$, Yeh SD, Yeung $H$, et al.Thallium-201 scintigraphy for the evaluation of tumor response to preoperative chemotherapy in patients with osteosarcoma. Cancer 1997;80:1507-1512. 